\title{
Abordagem fonoaudiológica na intervenção precoce em crianças com transtorno do espectro autista: revisão integrativa
}

Speech therapy approach in early intervention in children with autistic spectrum disorder: integrative review

Enfoque de la terapia del habla en la intervención temprana en niños con trastorno del espectro autista: revisión integrativa

Lucrecya Cena da Silva ORCID: https://orcid.org/0000-0002-2887-9446 FATEPI/FAESPI, Brasil

E-mail: lucrecyacena@outlook.com

Kamila Lopes de Lira ORCID: https://orcid.org/0000-0003-3267-6390 FATEPI/FAESPI, Brasil

E-mail: kmilalopes_17@ hotmai.com

Ruth Raquel Soares de Farias ORCID: https://orcid.org/0000-0002-0988-0900 FATEPI/FAESPI, Brasil

E-mail: ruthraquelsf@gmail.com

\begin{abstract}
Resumo
Este estudo objetiva identificar como o profissional fonoaudiólogo auxilia na intervenção precoce de crianças autistas. Trata-se de uma revisão integrativa da literatura, de abordagem quantitativa, qualitativa e descritiva. A coleta de dados ocorreu no período de setembro a novembro de 2021, foram coletados nas bases de dados: Biblioteca Virtual em Saúde (BVS), Portal Capes, PubMed, Scientific Electronic Library Online (SciELO). Para a pesquisa, foram usados os seguintes descritores: Ciência da Fala e Audição; Autismo Infantil; Fonoaudiologia. Os resultados abordam uma análise dos artigos em língua vernácula sobre as intervenções fonoaudiológicas no autismo. Ao todo, oito artigos atenderam aos critérios de inclusão e exclusão. Sendo 4 artigos da Scielo, 2 da CAPES e 2 da BVS. Conclui-se que a intervenção precoce no TEA é o que de melhor pode ser feito ao paciente e o quanto antes for iniciado maior é a chance de um controle sobre o desenvolvimento social e de comunicação da criança. O profissional fonoaudiólogo deve ser capaz de desenvolver na criança autista habilidades comunicativas, pois elas contribuem para a promoção da aprendizagem. Dessa forma, o fonoaudiólogo precisa estar atento para interpretar e contextualizar os processos de intervenção para que sua aplicação seja flexível e beneficie o paciente.
\end{abstract}

Palavras-chave: Ciência da fala e audição; Autismo infantil; Intervenções fonoaudiológicas.

\begin{abstract}
This study aims to identify how the speech therapist professional helps in the early intervention of autistic children. This is an integrative literature review, with a quantitative, qualitative and descriptive approach. Data collection took place from September to November 2021, and were collected in the following databases: Virtual Health Library (VHL), Portal Capes, PubMed, Scientific Electronic Library Online (SciELO). For the research, the following descriptors were used: Speech and Hearing Science; Child Autism; Speech Therapy. The results address an analysis of articles in vernacular language on speech therapy interventions in autism. In all, eight articles met the inclusion and exclusion criteria. With 4 articles from Scielo, 2 from CAPES and 2 from the VHL. It is concluded that early intervention in ASD is the best thing that can be done to the patient and the sooner it is started, the greater the chance of controlling the child's social and communication development. The speech therapist professional must be able to develop communication skills in autistic children, as they contribute to the promotion of learning. Thus, the speech therapist needs to be attentive to interpret and contextualize the intervention processes so that their application is flexible and benefits the patient.
\end{abstract}

Keywords: Speech and hearing science; Child autism; Speech therapy interventions.

\section{Resumen}

Este estudio tiene como objetivo identificar cómo el profesional del logopeda ayuda en la intervención temprana de niños autistas. Se trata de una revisión de la literatura integradora, con un enfoque cuantitativo, cualitativo y 
descriptivo. La recolección de datos se realizó de septiembre a noviembre de 2021, y se recolectaron en las siguientes bases de datos: Biblioteca Virtual en Salud (BVS), Portal Capes, PubMed, Scientific Electronic Library Online (SciELO). Para la investigación se utilizaron los siguientes descriptores: Ciencias del habla y la audición; Autismo infantil; Terapia del lenguaje. Los resultados abordan un análisis de artículos en lengua vernácula sobre intervenciones de logopedia en el autismo. En total, ocho artículos cumplieron los criterios de inclusión y exclusión. Con 4 artículos de Scielo, 2 de CAPES y 2 de la BVS. Se concluye que la intervención temprana en TEA es lo mejor que se le puede hacer al paciente y cuanto antes se inicie, mayores serán las posibilidades de controlar el desarrollo social y comunicativo del niño. El profesional del logopeda debe ser capaz de desarrollar habilidades comunicativas en niños autistas, ya que contribuyen a la promoción del aprendizaje. Así, el logopeda debe estar atento a interpretar y contextualizar los procesos de intervención para que su aplicación sea flexible y beneficie al paciente.

Palabras clave: Ciencias del habla y la audición; Autismo infantil; Intervenciones de logopedia.

\section{Introdução}

O Transtorno do Espectro Autista (TEA) é caracterizado como uma desordem do neurodesenvolvimento infantil, marcada por alterações sociocomunicativas e comportamentais (Kasari, 2016). O diagnóstico é feito por meio da díade: (a) déficit na interação social e comunicação e (b) comportamentos e interesses restritos e repetitivos (Apa, 2014).

O estudo mais recente do Centro de Controle e Prevenção de Doença (CDC), datado de 2020, estima que a prevalência do Transtorno do Espectro Autista é de 1 pessoa com autismo para cada 54 crianças. Os sintomas englobam déficits no funcionamento do cérebro da criança em desenvolvimento, ocasionando atraso na fala, na aprendizagem e na obtenção dos gestos motores (Soares \& Cavalcante Neto, 2015).

O tratamento padrão-ouro para o TEA é a intervenção precoce e consiste em um conjunto de modalidades terapêuticas que tem como objetivo aumentar o potencial do desenvolvimento social e de comunicação da criança, bem como proteger o funcionamento intelectual reduzindo danos, melhorar a qualidade de vida e dirigir competências para autonomia (Anagnostou et al., 2014).

As modalidades de intervenções diagnósticas para o TEA são: Modelo Denver de Intervenção Precoce para Crianças Autistas, que consiste em estimulação intensiva e diária baseada em Análise do Comportamento Aplicada (ABA), visando promover interações sociais positivas e naturalistas; Estimulação Comportamental baseada em ABA que consiste em um programa comportamental amplamente utilizado e reconhecido, que visa desenvolver habilidades sociais e comunicativas; Método TEACCH (Tratamento e Educação para Crianças Autistas e com outros prejuízos na comunicação), que estrutura o ambiente pedagógico-terapêutico com o estabelecimento de rotinas e o planejamento da sequência e duração das atividades (Cardoso et al., 2019).

$\mathrm{Na}$ literatura médica é consenso que, quanto mais cedo forem reconhecidas as alterações no desenvolvimento e comportamento da criança, no que diz respeito à sua história de vida afetiva, social e escolar, mais precoce poderá ser a intervenção e melhores serão os resultados (Cardoso et al., 2019).

A ausência de linguagem e aparente desinteresse pelo contato social podem ser características facilmente associadas ao TEA, por isso, uma avaliação audiológica deve diferenciar os déficits auditivos e o acompanhamento com um fonoaudiólogo contribui para um diagnóstico precoce e preciso. Por vezes, um período curto de terapia fonoaudiológica focada na interatividade possibilita tal diagnóstico (Fernandes, 2017).

O estudo do Transtorno do Espectro Autista é indiscutivelmente importante para a sociedade. Através da literatura científica é possível conhecer e estudar sobre os métodos de intervenção precoce. O profissional fonoaudiólogo que conhece e sabe aplicar os métodos de intervenção precoce na criança autista possui um papel diferencial no diagnóstico preciso e no cuidado com o paciente. Dessa forma, esta pesquisa pretende responder ao seguinte problema: Como o fonoaudiólogo pode colocar em prática os métodos de intervenção precoce do TEA?

A importância de conhecer os métodos de intervenção precoce do TEA vai além da conclusão diagnóstica. Porque, 
mesmo com o aumento dos casos de detecção muito ainda precisa ser feito para diminuir a falta de conhecimento por grande parte das pessoas - pais e profissionais de saúde - que precisam estar atentos aos seus filhos e pacientes. Por isso, faz-se necessário conhecer os métodos de intervenção precoce do TEA e entender como colocá-los em prática.

Diante dessa perspectiva, esta pesquisa busca como objetivos geral identificar como o profissional fonoaudiólogo auxilia na intervenção precoce de crianças autistas. E possui como objetivos específicos descrever a utilização prática dos métodos e/ou estratégias de intervenção; comparar os modelos e/ou estratégias de intervenção encontrados; e analisar os benefícios das estratégias de intervenção sob a perspectiva fonoaudiológica.

\section{Metodologia}

Este estudo trata-se de uma revisão integrativa da literatura, de abordagem quantitativa, qualitativa e descritiva. Escolhida por se tratar de uma abordagem metodológica ampla que permite realizar a síntese atual sobre determinada temática específica (Souza; Silva; Carvalho, 2010). O desenvolvimento do estudo deu-se primeiramente através da pergunta norteadora: Como o fonoaudiólogo pode colocar em prática os métodos de intervenção precoce do TEA?

Foi realizado uma busca da amostragem, por meio da coleta dos artigos científicos indexados em bases de dados confiáveis da área. Depois, os dados foram extraídos dos principais artigos originais que tratam sobre o tema para ser realizado a análise dos mesmos. Posteriormente, ocorreu a análise crítica do conteúdo dos estudos incluídos, por meio do rigor metodológico e as características de cada estudo. Foi realizada uma discussão dos dados coletados e, por fim, a apresentação da revisão integrativa.

A coleta de dados ocorreu no período de setembro a novembro de 2021, foram coletados os dados dos artigos científicos publicados nas bases de dados nacionais e internacionais: Biblioteca Virtual em Saúde (BVS), Portal Capes, PubMed, Scientific Electronic Library Online (SciELO). Considerando a confiabilidade das publicações na área da saúde nestas bases. Sendo utilizados os Descritores em Ciências da Saúde (DECS): Ciência da Fala e Audição; Autismo Infantil; Fonoaudiologia.

Os critérios de inclusão da pesquisa foram: artigos em português, disponibilizados na integra, referente aos últimos 10 anos de publicação (2011 - 2021), que retratassem a temática referente à esta revisão. Os critérios de exclusão foram: artigos cuja temática não se adequou ao tema proposto da pesquisa, artigos em outras línguas diferentes da vernácula, artigos com o ano de publicação fora do recorte temporal definido, os duplicados, e a literatura cinzenta: dissertações, monografias, capítulos de livros e editoriais.

Os seguintes dados foram extraídos das publicações: identificação, ano, objetivo, características metodológicas e os principais resultados de cada artigo, baseado no instrumento. A síntese dos dados se apresenta através de quadros para facilitar a visualização e compreensão dos resultados e discussão dos dados.

\section{Resultados e Discussão}

Por meio das pesquisas realizadas nas bases de dados, ao todo foram selecionados 40 artigos da base BVS, 50 da Scielo e 35 do CAPES, totalizando 125 artigos. Destes, 08 artigos confiáveis atenderam aos critérios de inclusão. Sendo 4 artigos da Scielo, 2 da CAPES e 2 da BVS.

Os resultados abordam uma análise dos artigos que discorram sobre as intervenções fonoaudiológicas de linguagem no TEA. Diante da leitura cautelosa, os estudos foram comparados, conforme o Quadro 1, que possui as seguintes informações: autor, ano, objetivos, metodologia e resultados de cada artigo selecionado. 
Quadro 1 - Levantamento da pesquisa com relação à base de dados, autores e ano, objetivos, metodologia e resultados.

\begin{tabular}{|c|c|c|c|}
\hline $\begin{array}{c}\text { Autores e } \\
\text { Ano }\end{array}$ & Objetivo & Metodologia & Resultados \\
\hline $\begin{array}{c}\text { Nunes e } \\
\text { Brito, } 2010\end{array}$ & $\begin{array}{l}\text { Descrever o processo de } \\
\text { intervenção fonoaudiológica } \\
\text { de dois irmãos com transtornos } \\
\text { invasivos do desenvolvimento, } \\
\text { por meio de um estudo } \\
\text { longitudinal de caso clínico. }\end{array}$ & $\begin{array}{l}\text { Participaram dois irmãos, um de nove e outro de } 11 \text { anos } \\
\text { de idade, ambos do gênero masculino, com autismo e } \\
\text { transtorno invasivo do desenvolvimento sem outra } \\
\text { especificação. Na coleta e análise de dados foi realizado } \\
\text { um estudo longitudinal, por meio de acompanhamento } \\
\text { dos casos ao longo de quatro anos de intervenção } \\
\text { fonoaudiológica. }\end{array}$ & 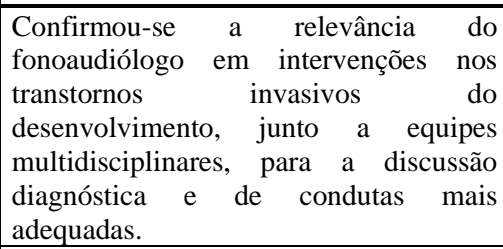 \\
\hline $\begin{array}{l}\text { Gonçalves e } \\
\text { Castro, } \\
2013\end{array}$ & $\begin{array}{l}\text { Revisar artigos atuais sobre } \\
\text { propostas de intervenção } \\
\text { fonoaudiológica no autismo } \\
\text { infantil, por meio de literatura } \\
\text { especializada. }\end{array}$ & $\begin{array}{l}\text { Pesquisa bibliográfica utilizando os bancos de dados } \\
\text { eletrônicos Medline, Lilacs e SciELO (2006-2010). As } \\
\text { palavras-chaves utilizadas em inglês foram: "autistic } \\
\text { disorder" e "speech therapy" nas bases de dados } \\
\text { Medline e Lilacs e em português: "autismo" e } \\
\text { "fonoaudiologia" na base de dados SciELO. }\end{array}$ & $\begin{array}{l}\text { Ao comparar os modelos e/ou } \\
\text { estratégias } \\
\text { fonoaudiológica, observou-se que eles } \\
\text { podem diferir não apenas entre } \\
\text { estruturados ou não estruturados, mas } \\
\text { também em relação ao objetivo, ao } \\
\text { modo ou forma de serem empregados, } \\
\text { possibilitando que a família e os } \\
\text { terapeutas possam escolher qual o } \\
\text { modelo adequado ao caso. Esse também } \\
\text { foi um fator encontrado nos textos, há } \\
\text { crescente preocupação quanto ao bem } \\
\text { estar da criança e da sua família. }\end{array}$ \\
\hline $\begin{array}{l}\text { Tamanaha, } \\
\text { Chiari e } \\
\text { Perissinoto, } \\
2015\end{array}$ & $\begin{array}{l}\text { Avaliar a eficácia da } \\
\text { intervenção terapêutica } \\
\text { fonoaudiológica para crianças } \\
\text { com Distúrbios do Espectro do } \\
\text { Autismo. }\end{array}$ & $\begin{array}{l}\text { A amostra foi composta por } 11 \text { crianças, divididas em } \\
\text { dois grupos: seis estavam recebendo intervenção direta e } \\
\text { indireta e cinco atendimento exclusivamente indireto. } \\
\text { Foram utilizados testes ASIEP-2: Autism Behavior } \\
\text { Checklist, Avaliação de Interação e Amostra do } \\
\text { Comportamento Vocal em três ocasiões: no início, seis } \\
\text { meses depois, e após } 12 \text { meses. }\end{array}$ & $\begin{array}{l}\text { Observou-se maior evolução do GT no } \\
\text { Autism Behavior Checklist, Avaliação } \\
\text { Interação e na Amostra de } \\
\text { comportamento vocal. Tanto as mães } \\
\text { quanto a fonoaudióloga perceberam } \\
\text { mudanças comportamentais. }\end{array}$ \\
\hline $\begin{array}{l}\text { Pedruzzi e } \\
\text { Almeida, } \\
2018\end{array}$ & $\begin{array}{l}\text { Verificar a proposta do jogo } \\
\text { simbólico na intervenção } \\
\text { fonoaudiológica com crianças } \\
\text { diagnosticadas com Transtorno } \\
\text { do Espectro Autístico. }\end{array}$ & $\begin{array}{l}\text { Estudo de casos, com abordagem qualitativa. Foi } \\
\text { realizada em duas instituições filantrópicas de uma } \\
\text { capital de um estado do Nordeste, com fonoaudiólogos } \\
\text { que atuam com crianças autistas. Os fonoaudiólogos, } \\
\text { denominados como F1, F2, F3, F4 e F5, responderam a } \\
\text { uma entrevista estruturada com treze questões. } \\
\end{array}$ & $\begin{array}{l}\text { Observou-se maior evolução do GT no } \\
\text { Autism Behavior Checklist, Avaliação } \\
\text { Interação e na Amostra de } \\
\text { comportamento vocal. Tanto as mães } \\
\text { quanto a fonoaudióloga perceberam } \\
\text { mudanças comportamentais. } \\
\end{array}$ \\
\hline $\begin{array}{c}\text { Cruz, } \\
\text { Gomes e } \\
\text { Lacerda, } \\
2020\end{array}$ & 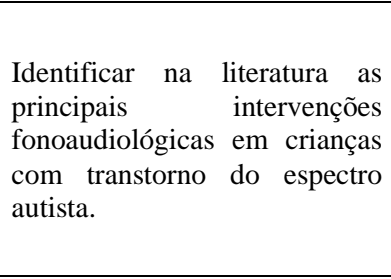 & $\begin{array}{l}\text { Trata-se de uma revisão bibliográfica integrativa da } \\
\text { literatura, os critérios definidos para inclusão foram: } \\
\text { publicações datadas de } 2010 \text { a } 2020 \text { com relação direta } \\
\text { com o assunto pesquisado, foram selecionados nove } \\
\text { artigos relacionados ao tema. }\end{array}$ & $\begin{array}{l}\text { Parte das pesquisas evidenciadas } \\
\text { demonstraram que a associação da } \\
\text { terapia lúdica com o fonoaudiólogo } \\
\text { assumindo papel de interlocutor, } \\
\text { proporciona uma intervenção satisfatória } \\
\text { que possibilitam evoluçâo considerável } \\
\text { no desenvolvimento do sujeito com } \\
\text { TEA. }\end{array}$ \\
\hline $\begin{array}{l}\text { Cardoso et } \\
\text { al., } 2020\end{array}$ & $\begin{array}{l}\text { Compreender como a } \\
\text { utilização da estratégia da roda } \\
\text { de conversa como forma de } \\
\text { intervenção junto às famílias } \\
\text { de crianças com diagnóstico de } \\
\text { alteraça de linguagem auxilia } \\
\text { no processo de } \\
\text { desenvolvimento comunicativo } \\
\text { destes indivíduos. }\end{array}$ & $\begin{array}{l}\text { Participaram vinte e dois pais e ou cuidadores de } \\
\text { crianças com diagnóstico de alteração de linguagem } \\
\text { divididos em dois grupos: Gagueira Desenvolvimental } \\
\text { Persistente (GDP) e Transtorno do Espectro Autista } \\
\text { (TEA) em atendimento fonoaudiológico. }\end{array}$ & $\begin{array}{l}\text { Algumas nuances quanto ao discurso } \\
\text { podem ser evidenciadas, principalmente } \\
\text { quando encontramos uma temática } \\
\text { semelhante: a fala. Os relatos destacam a } \\
\text { necessidade de compreender a } \\
\text { competência comunicativa da criança e } \\
\text { o impacto das alterações no processo de } \\
\text { socialização, ou seja, a compreensão do } \\
\text { outro desta comunicação e suas falhas. }\end{array}$ \\
\hline $\begin{array}{l}\text { Araújo et } \\
\text { al., } 2021\end{array}$ & $\begin{array}{l}\text { Analisar a importância das } \\
\text { intervenções fonoaudiológicas } \\
\text { em crianças autistas. }\end{array}$ & $\begin{array}{l}\text { Para a fundamentação da pesquisa houve a necessidade } \\
\text { de uma revisão bibliográfica do tipo integrativa, } \\
\text { anlisando diversas fontes, presentes em artigos } \\
\text { científicos, disponíveis nas bases de dados da Scielo, } \\
\text { Pubmed, Google Acadêmico e Capes. }\end{array}$ & $\begin{array}{l}\text { Foi evidenciado por meio da pesquisa } \\
\text { que são eficazes no desenvolvimento da } \\
\text { criança autista possibilitando um maior } \\
\text { benefício no desenvolvimento } \\
\text { comunicativos desses seres humanos. } \\
\end{array}$ \\
\hline $\begin{array}{l}\text { Silva e } \\
\text { Coelho, } \\
2021\end{array}$ & $\begin{array}{l}\text { Apresentar levantamento de } \\
\text { publicações cientificas sobre a } \\
\text { aquisição da linguagem de } \\
\text { crianças do transtorno do } \\
\text { espectro autista, abordando as } \\
\text { intervenções utilizados pelos } \\
\text { fonoaudiólogos. }\end{array}$ & $\begin{array}{l}\text { É uma revisão de literatura crítica, de análise qualitativa, } \\
\text { que tem o intuito de sistematizar resultados de artigos, } \\
\text { teses e resultados de pesquisas realizadas com o } \\
\text { processo de aquisição de linguagem em indivíduos com } \\
\text { transtorno do Espectro Autista e o fonoaudiólogo como } \\
\text { profissional relevante neste processo. }\end{array}$ & $\begin{array}{l}\text { Os estudos encontrados completam } \\
\text { vários aspectos no processo de aquisição } \\
\text { da linguagem, estratégias e práticas que } \\
\text { favorecem esse processo, escola e } \\
\text { profissionais com intervenções com base } \\
\text { na singularidade de cada sujeito, } \\
\text { favorecendo a inserção social dos } \\
\text { autistas em meios comunicativos e } \\
\text { conversacionais. }\end{array}$ \\
\hline
\end{tabular}


Conforme destaca Cruz, Gomes e Lacerda (2020), o brincar é um elemento chave para o avanço da linguagem em crianças, visto que possibilita o desenvolvimento e a inserção desta na língua. As autoras destacam ainda que as atividades lúdicas permitirão o reconhecimento e trocas com o outro, no qual esse contexto lúdico fornece situações sem o peso de punições, possibilitando que a criança se sinta confortável. É salutar discutir que o brincar para a criança autistas não é um processo simples, pode ser longo e trazer grandes frustrações a pais, familiares e educadores, que acabam desacreditando da sua viabilidade e importância para o desenvolvimento da criança. Dessa forma, Martins e De Goés (2013) corroboram com as autoras supracitadas quando afirmam em seu estudo que a característica central do brincar está no fato de que a criança aprende a agir no campo da significação ao invés de se restringir ao campo perceptual, dependendo mais de suas motivações que das características dos objetos externos. Assim, o campo percebido perde sua força determinante e a criança passa a agir de maneira diversa frente àquilo que vê, operando com significados libertos das características dos objetos e das ações às quais estes estão habitualmente vinculados.

Pedruzzi e Almeida (2018), em seu estudo, ressaltam que crianças com TEA apresentam peculiaridades e necessidades distintas; porém é notável o déficit na capacidade de simbolizar. A estimulação do jogo simbólico quando inserida no processo terapêutico, visa estimular a intenção comunicativa, resolução de problemas para promover o desenvolvimento da linguagem e da interação social.

Os autores Silva e Coelho (2021) em sua pesquisa bibliográfica, afirmam que a Fonoaudiologia se ocupa da habilidade de desenvolver a comunicação humana, e no autismo provoca melhorias nas habilidades comunicativas, que contribui para a promoção da aprendizagem. Ressaltam ainda que a consciência fonológica é fator decisivo para a aprendizagem da escrita, sendo um importante estímulo antes do ciclo de alfabetização. Nesse sentido, Freire (2018) afirma que o fonoaudiólogo é o profissional que visa em suas intervenções a melhoria dos sintomas relacionado a linguagem e comunicação, seja ela verbal ou não - verbal. Por isso, uma intervenção precoce e continua auxilia a evolução e desenvolvimento da criança, conforme o programa terapêutico específico.

Através da pesquisa realizada por Gonçalves e Castro (2013) sobre uma proposta de intervenção precoce no autismo infantil, é possível destacar dois pontos importantes. O primeiro é que os diferentes modelos de intervenção precoce refletem a preocupação com a individualidade da criança, ou seja, a percepção de que eles têm características em comum, mas cada um tem as suas particularidades; e é por isso que os métodos mais eficazes são os baseados no binômio (profissional e criança), através do modelo de 1 para 1. Outro ponto é que o profissional fonoaudiólogo precisa estar atento para interpretar e contextualizar os processos de intervenção para que sua aplicação seja flexível e beneficie o paciente; por isso, o conhecimento acerca dos métodos e a melhor forma de aplicá-los é a base para um tratamento eficaz. E assim como destaca Araújo et al. (2021) a atuação dos fonoaudiólogos - intervindo direta e indiretamente - possibilita uma evolução mais ampla no que se refere ao desenvolvimento de crianças com transtornos do espectro autista, o que favorece um ambiente enriquecedor e aberto para a presença dos pais ou responsáveis.

Quando à relação terapeuta-paciente, no estudo de Nunes e Brito (2010), os autores estabelecem a importância do contato visual, da atenção conjunta, da imitação e jogo compartilhado, que têm como objetivo desenvolver a competência comunicativa com ênfase no uso funcional da linguagem. Já o estudo de alvarenga (2017) traz uma abordagem terapêutica de decodificação verbal das condutas não verbais e ensina o indivíduo a dirigir sua atenção a todo esse processo, chamado de terapia cognitivo-comportamental. O estudo conclui com a afirmação de que esse modelo de intervenção facilita a inabilidade do paciente de se socializar e a dificuldade de se comunicar. Utilizando-se do treino de habilidades sociais, que envolve estratégias de reestruturação cognitiva, e técnicas comportamentais, por meio do processo de atenção terapêutica.

Outro estudo que destaca o tratamento terapêutico é o de Tamanaha, Chiari e Perissinoto (2015), os autores evidenciam que nos primeiros seis meses de vida da criança a extensão e a velocidade do processo evolutivo tornaram-se mais 
evidentes, pincipalmente nas crianças assistidas pelo método direto, ou seja, durante o primeiro semestre as orientações e a própria atuação direta com a criança causaram um impacto maior, permitindo um ganho terapêutico mais expressivo. O autor Bosa (2006) corrobora com o autor supracitado, pois segundo ele, o tratamento com crianças pequenas, deveria ser a terapia da fala, da interação social/linguagem, educação especial e suporte familiar.

\section{Conclusão}

A intervenção precoce no TEA é o que de melhor pode ser feito ao paciente, visto que as medidas de prevenção são as mais eficazes quando se trata de diminuir os efeitos deletérios do autismo, ou seja, quanto antes for iniciado maior é a chance de um controle sobre o desenvolvimento social e de comunicação da criança.

O profissional fonoaudiólogo deve ser capaz de desenvolver na criança autista habilidades comunicativas, pois elas contribuem para a promoção da aprendizagem. Dessa forma, o fonoaudiólogo visa em suas intervenções a melhoria dos sintomas relacionado a linguagem e comunicação, seja ela verbal ou não verbal. E é por isso que uma intervenção precoce auxilia na evolução e desenvolvimento da criança, conforme o programa terapêutico específico.

Da mesma forma, o fonoaudiólogo precisa estar atento para interpretar e contextualizar os processos de intervenção para que sua aplicação seja flexível e beneficie o paciente; por isso, o conhecimento acerca dos métodos e a melhor forma de aplicá-los é a base para um tratamento eficaz. Um profissional capacitado favorece a criação de um ambiente enriquecedor e aberto para a presença e contribuição dos pais ou responsáveis no processo terapêutico.

Sendo assim, considera-se essa temática fundamental para a atuação do fonoaudiólogo, bem como, nas intervenções práticas de métodos frente às crianças com TEA. Para tanto, sugere-se a continuidade de pesquisas, estudos e mais artigos enfatizando sobre o assunto, favorecendo assim, novos conhecimentos e possibilidades para o trabalho do profissional fonoaudiólogo.

\section{Referências}

Alvarenga, G. C. S. (2017). Autismo leve e intervenção na abordagem cognitivo-comportamental. Trabalho de conclusão de curso Lato Sensu.

American Psychiatric Association. (2014). Manual de diagnóstico e estatístico de transtornos mentais: DSM-V. Artmed. 848 p.

Anagnostou, E et al. (2014). Autism spectrum disorder: advances in evidence-based practice. CMAJ.;186(7):509-19.

APA (American Psychiatric Association). (2014). Transtornos mentais. DSM-V. In: APA (American Psychiatric Association), Manual diagnóstico e estatísticos de transtornos mentais. (5a ed.), Artmed.

Araújo, J. L. O et al. (2021). Benefícios da intervenção fonoaudiológica no transtorno do espectro autista: Revisão de literatura. Research, Society and Development, v. 10, n. 6, e49610615550. http://dx.doi.org/10.33448/rsd-v10i6.15550.

Bosa, C. A. (2006). Autismo: intervenções psicoeducacionais. Braz. J. Psychiatry 28 (suppl 1). https://doi.org/10.1590/S1516-44462006000500007.

Cardoso, A. A et al. (2019). Transtorno do Espectro do Autismo. Sociedade Brasileira de Pediatria. № 05, abril.

Cardoso, C et al. (2020). Rodas de conversa e fonoaudiologia: estratégia de intervenção nas alterações de comunicação. Revista Brasileira de Geografia Médica e da Saúde. HYGEIA. http://www.seer.ufu.br/index.php/hygeia.

Cruz, B. P., Gomes, L. G. A. A., \& Lacerda, M. C. (2020). Intervenção fonoaudiológica em crianças com Transtorno do Espectro Autista. Trabalho de Conclusão de Curso. https://repositorio.pucgoias.edu.br/jspui/handle/123456789/436

Silva. W. M. da., \& Coelho, A. T. C. B. (2021). O processo de aquisiçao de linguagem para a criança com transtorno do espectro autista: artigo de revisão. Research, Society and Development, 10(1), e15010111584. http://dx.doi.org/10.33448/rsd-v10i1.11584.

Fernandes, F. (2017). Advances in Speechlanguage Pathology. InTech. https://www.intechopen.com/books/5957

Freire, T. (2018). Ações da fonoaudiologia na escola: programa de estimulação da consciência fonológica em escolares do 1 ano. (Tese de Doutorado) Universalidade de São Paulo. Faculdade de Odontologia de Bauru. Bauru, SP, Brasil.

Gonçalves, C. A. B., \& Castro, M. S. J. (2013). Propostas de intervenção fonoaudiológica no autismo infantil: revisão sistemática da literatura. Distúrb Comun, São Paulo, 25(1): 15-25. file://C:/Users/MULTILASER/Desktop/14920-Texto\%20do\%20Artigo-35920-1-10-20130427.pdf. 
Research, Society and Development, v. 10, n. 15, e583101523353, 2021

(CC BY 4.0) | ISSN 2525-3409 | DOI: http://dx.doi.org/10.33448/rsd-v10i15.23353

Kasari, C. (2016). Assessing Change in Early Intervention Programs for children with Austim.In: Journal of Autismand Developmental Disorders. 54(16).

Martins, A. D. F., \& De Góes, M. C. R. (2013). Martins Um estudo sobre o brincar de crianças autistas na perspectiva histórico-cultural. Revista Semestral da Associação Brasileira de Psicologia Escolar e Educacional, 17(1): 25-34.

Misquiatti, A. R. N., \& Brito, M. C. (2010). Terapia de linguagem de irmãos com transtornos invasivos do desenvolvimento: estudo longitudinal. Rev. soc. bras. fonoaudiol. 15(1). https://doi.org/10.1590/S1516-80342010000100022.

Oliveira, T. R. S et al (2018). Intervenção fonoaudiológica e autismo. Rev. CEFAC. 20(6), 808-814.

Paul, R., \& Norbury, C. (2012). Distúrbios da linguagem da infância à adolescência: ouvir, falar, ler, escrever e se comunicar. St. Louis, MO: Elsevier Health Sciences.

Pedruzzi, C. M., \& Almeida, C. H. A. A. (2018). O jogo simbólico na intervenção fonoaudiológica de crianças com transtorno do espectro autístico. Distúrb Comun, 30(2): 242-251. http://dx.doi.org/10.23925/2176-2724.2018v30i2p-242-251.

Soares, A. M., \& Cavalcante Neto, J. L. (2015). Avaliação do comportamento motor em crianças com transtorno do espectro do autismo: uma revisão sistemática. Rev. Brasileira de educação, 21(3), 445-458. https://doi.org/10.1590/S1413-65382115000300010.

Souza, M. T., Silva, M. D. \& Carvalho, R. (2010). Revisão integrativa: o que é e como fazer. Einstein 8 (1): 102-106.

Tamanaha, A. C., Chiari, B. M., \& Perissinoto, J. (2015). A eficácia da intervenção terapêutica fonoaudiológica nos distúrbios do espectro do autismo. Rev. CEFAC. 17 (2). https://doi.org/10.1590/1982-021620156314. 